\title{
Design of an Architecture for Optimizing Image Retrieval by using Genetic Algorithm
}

\author{
S.Selvam \\ M.Sc., M.Phil., \\ Research Scholar \\ Research and Development Center \\ Bharathiar University \\ Coimbatore- 641046 \\ Assistant Professor \\ Department of Computer Applications \\ N.M.S.S.Vellaichamy Nadar College, Madurai-19
}

\author{
S.Thabasukannan \\ MCA, M.Phil., MBA., M.Phil.,M.Tech., Ph.D, \\ Principal, Pannai College of Engg\&Tech \\ Sivagangai - 630 561, Tamilnadu, India
}

\begin{abstract}
Image retrieval plays a vital role in image processing. The main aim of this paper is to build more generalized CBIR system which is increased the searching ability and to improve the retrieval accuracy. The proposed method is experimental and analyzed with large database. The result show that the architecture of new CBIR system shown good performance in speed and reducing the computational time.
\end{abstract}

\section{Keywords}

CBIR, Genetic Algorithm, HARP Algorithm, Precision, Recall

\section{INTRODUCTION}

The enormous collection of digital images on personal computers, institutional computers and Internet necessitates the need to find a particular image or a collection of images of interest. This has motivated many researchers to find efficient, effective and accurate algorithms that are domain independent for representation, description and retrieval of images of interest. There have been many algorithms developed to represent, describe and retrieve images using their visual features such as shape, color and texture. The visual feature representation and description play an important role in image classification, recognition and retrieval. The content based image similarity measurement algorithms, if chosen correctly for a particular image representation technique, will definitely increase the efficiency and effectiveness retrieval of data of interest.

Nowadays national geographic imagery archive has a size of Petabytes (PB) and grows to Terabytes (TB). It triggers the demand of qualitative and quantitative image retrieval systems. An image retrieval system is a computer based system for browsing, searching and retrieving images from a large database of digital images. Searching and retrieving is not bit by bit comparison. It is not a matching process on the raw data.

The drawbacks of the TBIR initiate to do the research in the field of CBIR. In CBIR also known as query by image content (QBIC), retrieval is based on the image contents. Many techniques have been developed for the most important CBIR systems, which is a system, in which retrieves visual-similar images from large image database based on automatically derived image features, which has been a very active area recently. In most of the existing CBIR systems, the image content is represented by their low-level features such as colour, texture and shape. The drawback of low-level features is losing much detail information of the images, in case of looking for images that contain the same object or same scene with different viewpoints. In recent years, the interest point detectors and descriptors are employed in many CBIR systems to overcome the above drawback.

Similarity can be defined as the quantitative measurement that indicates the strength of relationship between two image objects. Dissimilarity is also a quantitative measurement that reflects the discrepancy between two image objects.

In a CBIR system, the retrieval of images has been done by similarity comparison between the query image and all candidate images in the database. To evaluate the similarity between two images, the simplest way is to calculate the distance between the feature vectors representing the two images. To find more similar or relative images, the heuristic approach based Genetic algorithm has been used in the CBIR system.

Image retrieval techniques are useful in many imageprocessing applications. Content-based image retrieval systems work with whole images and searching is based on comparison of the query. General techniques for image retrieval are color, texture and shape. These techniques are applied to get an image from the image database. They are not concerned with the various resolutions of the images, size and spatial color distribution. The content and metadata based system gives images using an effective image retrieval technique.

The primary goal of this paper is to reduce the computation time and user interaction. The conventional CBIR systems also display the large amount of results at the end of the process this will drove the user to spend more time to analyze the output images. In the proposed system we compute texture feature and color feature for compute the similarity between query and database images. This integrated approach will reduce the output results to a certain levels based on the user threshold value. The secondary goal is to reduce semantic gap between high level concepts and low level features. Generally the CBIR compute the similarity between the query image and the database images. Hence there might be chances for unexpected results at the end the retrieval process. The novel 
HARP cluster the output images and select one representative image from each clusters. A third goal is to evaluate their performance with regard to speed and accuracy. These properties were chosen because they have the greatest impact on the implementation effort

Instead of Relevance Feedback we can use any clustering algorithm that based on the features extracted from the images themselves, and allocates those images into the nearest cluster. The algorithm calculates sand allocates until there is little variation in the movement of feature points in each cluster. Clustering is the unsupervised classification of patterns into groups. Its main task is to assigning a set of objects into groups so that the objects in the same cluster are more similar to each other than to those in other clusters.

In this paper, Color, Texture and Shape features were extracted and combined to form feature vector of image. For color features, the moments of the color distribution were calculated from the images and used as color descriptor. For texture features, we used Gabor filter, which is a powerful texture extraction technique in describing the content of image. For shape features, edge histogram features that include five categories were used as shape descriptor. These three descriptors were combined and optimized using GA with HARP clustering accuracy as a fitness function to select optimum weights of features. We performed GA with HARP clustering on the database as an offline step, and the system does not need to search the entire database images; instead just a number of candidate images are required to be searched for image similarity

\section{PREVIOUS STUDY}

There are various approaches are present for CBIR. Some of the important literature which covers the more important CBIR System is discussed below.

i Chin-Chin Lai et.al. have proposed an interactive genetic algorithm (IGA) to reduce the gap between the retrieval results and the users' expectation. They have used color attributes like the mean value, standard deviation, and image bitmap. They have also used texture features like the entropy based on the gray level co-occurrence matrix and the edge histogram.

ii Zhang Xu-bo et.al.have published a paper on improved $\mathrm{K}$-means clustering and relevance feedback to re-rank the search result in order to remedy the rank inversion problem in CBIR. Experimental results show that the reranking algorithm achieves a more rational ranking of retrieval results and it is superior to Reranking via partial Grouping method

iii Lijun Zhao et.al.have proposed a multi-round relevance feedback (RF) strategy based on both support vector machine (SVM) and feature similarity to reduce the gap between query and retrieve result.

iv SharadhRamaswamy et.al.have published a paper on a fast clustering-based indexing technique. In this method relevant clusters are retrieved till the exact nearest neighbors are found. This enables efficient clustering with low preprocessing storage and computation costs.

v Nhu-Van Nguyen et.al. have proposed Clustering and Image Mining Technique for fast Retrieval of Images. The main objective of the image mining is to remove the data loss and extracting the meaningful information to the human expected needs. The clustering-repeat gives good result when the number of examples of feedback is small.

vi Hua Yuan et.al. have presented a new statistical modelbased image feature extraction method in the wavelet domain and a novel Kullback divergence-based similarity measure. The Gaussian Mixture Models(GMM) and Generalized GMM are presented to help extract new image features.From the literature survey it is concluded that a wide variety of CBIR algorithms have been proposed in different papers. The selection feature is one of the important aspects of Image Retrieval System to better capture user's intention. It will display the images from database which are the more interest to the user.

\section{ARCHITECTURE OF NEW CBIR SYSTEM}

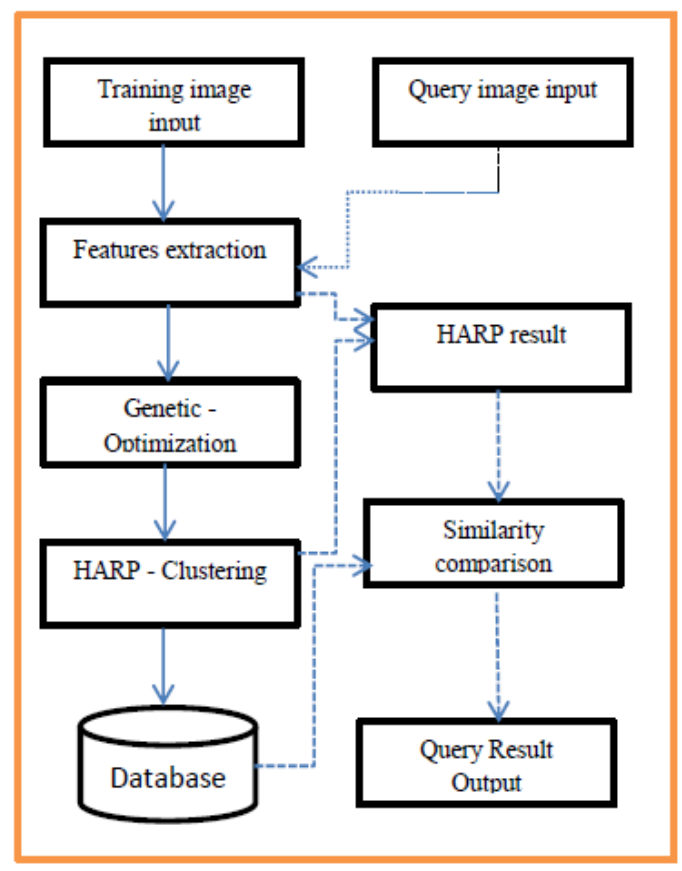

Figure 1: A New architecture for CBIR

Training Image Input: The learning phase tells about the training process which a huge amount sample images are input in the first step. The genetic algorithm is used to train the features with different weights. For optimizing the feature weights and for fitness function, HARP algorithm is used. The training part outputs the classifying result and stores it in the feature database. All these steps performed offline and each class will be indexing along with its associated classID in the index files.

Feature Extraction (Image signature): There are various kinds of visual features to represent an image, such as color, texture, shape, and spatial relationship. Since one type of features can only represent part of the image properties, a lot of work done on the combination of these features. The feature of each image is very much smaller in size compared to the image data, so the feature database contains an abstraction of the images in the image database.

i Colors: Are defined on a selected color space. Varieties of color spaces include, RGB, LAB, LUV, HSV (HSL), $\mathrm{YCrCb}$ and the HMMD. Common color features or 
descriptors in CBIR systems includecolor-covariance matrix, color histogram, color momentsand color coherence vectorstoring, filtering and retrieving audiovisual data. The emerging MPEG-7 is a new multimedia standard, which has improved CBIR by providing a rich set of standardized descriptors and description schemas for describing multimedia content. MPEG-7 has included dominant color, color structure, scalable color, and color layout as color features. Here we used Color Structure Descriptor (CSD) as color feature.The CSD represents an image by both the color distribution of the image or image region and the local spatial structure of the color. CSD used a $8 \times 8$ structure to scan the total image. This descriptor counts the number of times a particular color is contained within the structuringelement while the image or image region is scanned by this structuring element.It has used HMMD color space.

ii Texture: There exist different approaches to extract and represent textures. They can be classified into spacebased, frequency-based models, and texture signatures. Some popular techniques i.e wavelet transform, cooccurrence matrix, and Gabor filters are applied to express texture features for image.

iii Shape: It is seen that natural objects are primarily recognized by their shape. Two main types of shape feature are commonly used; global features such as aspect ratio, circularity and moment invariants and local features such as sets of consecutive boundary segments.

Genetic Algorithm: For optimization:It is used to find solution to complicated problems. It is based on heuristic approach that imitates the process of natural selection. It is used exclusively for the purpose of optimization. Each intermediary solution can be mutated and changed. It contains the following operators:

Selection: Duringeachiterationexisting input iterations are refreshed through a fitness process. If any iteration is best fit, then it is the solution and no further selection is needed.

Mutation is an interchange of data from one iteration to another.

Cross over is a process n-parent solutions used to derive a child solution.

HARP-a Clustering algorithm: The algorithm is based on bottom-up approach. Initially pick up each element among all current clusters on the basis of smallest distance by merging all the selected and related data on various clusters.

In HARP algorithm, the accuracy level of clustering is more by using relevance indexing and merge score. The scalability level is also very high. The time taken for finding the closest cluster is very less.

Database: A database containing number of images with any one of the formats.bmp, .jpg, .tiff. is required.

Query: The user provides a sample image or sketched figure as the query for the system. This phase describes the images searching process. The user enters a query image for which the system extracts color, texture and shape features the features vectors of database images are previously extracted and stored.
Similarity Matching: Using the similarity metrics defined for color, texture and shape, the similarity distances between the query image and the centroid image of each class are calculated. The smallest distance (most similar) will determine to which the image belongs. The class with the smallest distance is returned and the images in this class will be compared with the query image.

Retrieval: The most matching images will be retrieved and then they are sorted in ascending order. The first $\mathrm{N}$ similar target images.

\section{PERFORMANCE EVALUATION}

Compare the new system results with some other existing CBIR systems. Large Image database is used in our evaluation. It is a sub set of the Corel database of 1,000 images in JPEG format.1,000 image database went through our implemented system to extract the features and stored them. The extracted features are weighted by GA and they are used for classification by using the HARP algorithm. The level of retrieval accuracy is a factor to influence the performance. In CBIR, the most commonly used performance measures are Precision and Recall. Precision is defined as the ratio of the number of retrieved relevant images to the total number of retrieved images. This means that precision measures the accuracy of the retrieval. Recall is defined as the ratio of the number of retrieved relevant images to the total number of relevant images in the database. The recall measures the robustness of the retrieval.

In CBIR, if the precision score is 1.0 then every image retrieved by a search is Relevant. If the recall score is 1.0 then all relevant images are retrieved by the search is robust. We evaluate the new system by using two metrics viz: the Retrieval Effectiveness and the Retrieval Efficiency.

i Retrieval Effectiveness: A retrieved image is considered a match if it is in the same class as the query image. The system works well and it retrieves better results over the randomly selected images as queries by using GA and HARP algorithm.

ii Retrieval Efficiency: By assigning different weights to each feature to improve the efficiency we have used GA with a HARP algorithm to select optimum weights of features to get the accuracy.

Here by using clustering pre-process of the database image via HARP algorithm decreases the average query response time, the similarity search time for image matching and increases the efficiency of the system.

\section{Comparison of the new system with other existing systems}

For each class in the database, randomly selected 20 images as queries. Since there are 3 classes in the database, it contains 60 query images. For each query, calculate the precision and recall of the retrieval. The average precisions and the average recall for each class based on the returned top 20 images were recorded. Moreover the new system result is compared against the performance of three methods. 


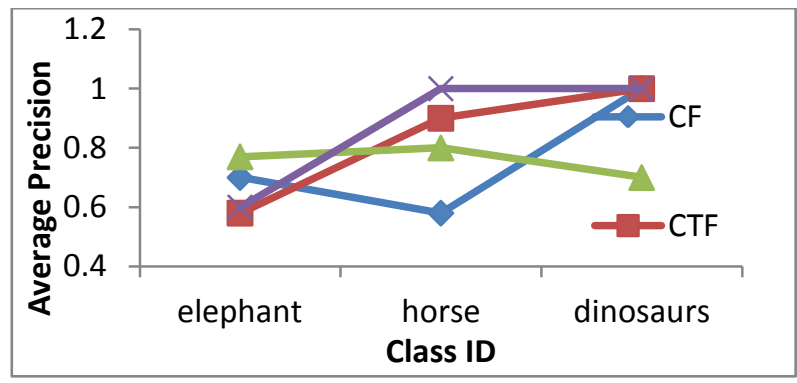

Figure 2: Comparison of Precision of the new system with various types of features.

The above figure shows that the new system performs significantly better in all three classes except elephant class. This result confirms that a fusion of multiple feature scan increase the performance of the system.

The below figure shows that the new system performs significantly better than other existing systems for all classes except elephant class. This is a good indicator for the effectiveness of our system. The reason behind the limitation in two classes is that those classes' images are very similar in term of the dominant color, texture and shape so, our new system may confused between them.

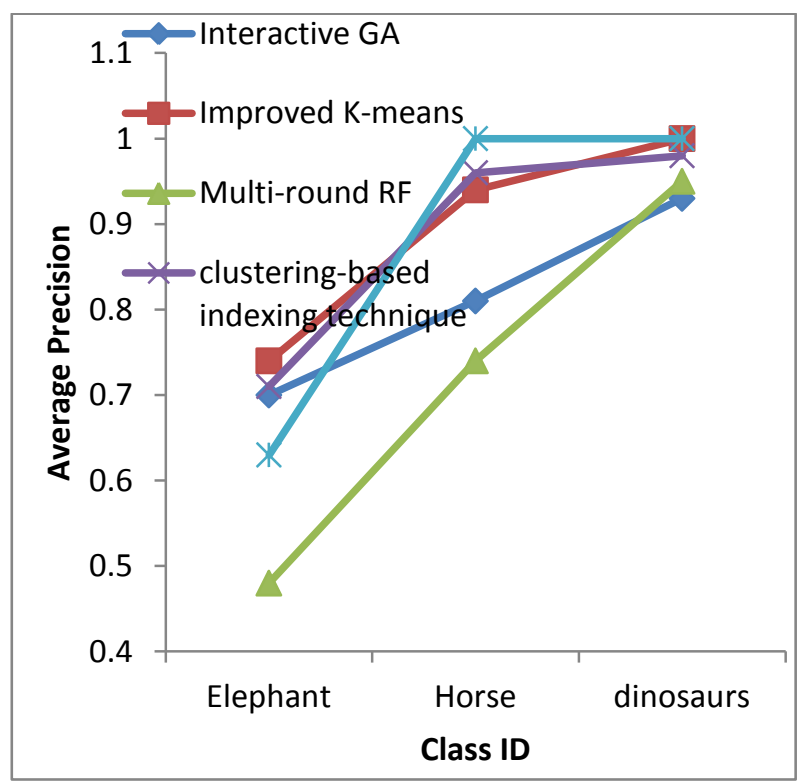

Figure3: Comparison of Precision of the new system with some existing Systems

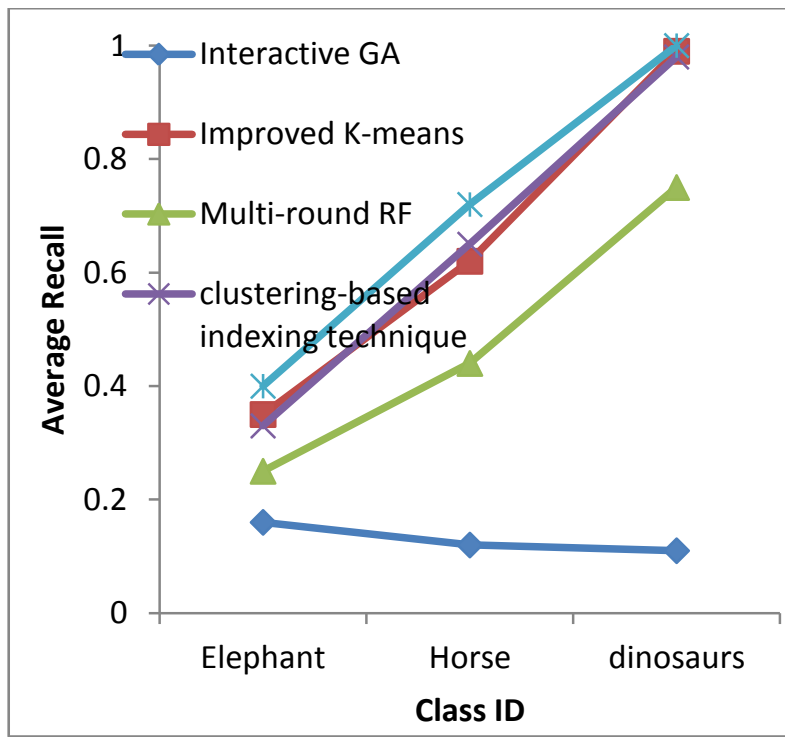

Figure4: Comparison of Recall of the new System with some existing systems

The above figure shows that the new system performs significantly better than other systems, for all classes. This means that the new system can retrieve most of database images that match query image. The new system works well in the classification part of using GA with HARP algorithm. The average precision and the average recall increased from $78.1 \%$ to $88.2 \%$ and $50.4 \%$ to $69.9 \%$ respectively and obtained an average reduction in 6.20 seconds.

\section{CONCLUSION}

The explosive growth of image data leads to the need of research and development of Image Retrieval.CBIR is currently a keen area of research in the area of multimedia databases. Various research works had been undertaken in the past decade to design efficient image retrieval techniques from the image databases. More précised retrieval techniques are needed to access the large image archives being generated, for finding relatively similar images. In this work the GA is combined with HARP clustering algorithm to improve the retrieval accuracy of the system. Getting lower computational time and retrieving relevant and accurate image is possible by using CBIR. In future we have a proposal to disseminate the features selections and use other distance measures to improve the overall results.

The efficiency of the new system is improved by considering candidate images for similarity computation purpose i.e. not considering the whole database images. A candidate image lies in the same cluster with the query image the benefit of the clustering process clearly proved the retrieval accuracy.

\section{REFERENCES}

[1] V.Gudivada and V.Raghavan, "Content-based Image Retrieval Systems," IEEE Computer, vol. 28, no 9, pp1822, Sep. 1995.

[2] F.Long,H.Zhang,H.Dagan,andD. Feng, "Fundamentals of Content Based Image Retrieval," Multimedia Signal Processing Book, Chapter1, Springer-Verlag, Berlin Heidelberg New York, 2003.

[3] S.Selvam and Dr.S.Thabasu Kannan, "Design of an Effective Method for Image Retrieval”, published 
IJIRAE, International Journal of Innovative Research in Advanced Engineering, Volume-1, March 2014, pp.5156.

[4] S.Selvam and Dr.S.Thabasu Kannan, "An Empirical Review on Image Retrieval System by using Relevance Feedback" proceeding of International Symposium on "Research innovation for quality improvement in Higher Education" conducted by Bharathiar University, Coimbatore, October 2014 and published in "Research and Trends in Data mining and Image Processing Technologies and Applications", Bloomsbury publishing India, London, New Delhi, New York, Sydney pp-1-11, October 2014, ISBN: 978-93-84052-11-9.

[5] R.Chang,J.Ho,S.Lin,C.FannandY.Wang,"ANovelConten tBasedImageRetrievalSystemusingK-means with Feature Extraction, "International Conference on Systems and Informatics, 2012.

[6] I.El-Naqa, Y.Yang, N.Galatsanos, R.Nishikawa and M.Wernick, "A Similarity Learning Approach to Content-Based Image Retrieval: Application to Digital Mammography," IEEE Transactions on Medical Imaging, 2009.

[7] B.WANG,X.ZHANG,andN.LI, "Relevance Feedback Technique For Content-Based Image Retrieval Using Neural Network Learning, "Proceedings of the Fifth International Conference on Machine Learning and Cybernetics, Dalian, 2006.
[8] R.Datta,J.Li,andJ.Wang,"Content-BasedImageRetrievalApproachesandTrends of theNew Age,"ACMComputing Surveys, vol. 40, no. 2, pp. 1-60,April 2008.

[9] J.HanandM.Kambr,"DataMiningConceptsandTechnique, "2ndEd.,MorganKaufmannPublisher, 2006.

[10] P.JeyanthiandV.JawaharSenthil Kumar, "Image Classification by K-means Clustering. "Advances in Computational Sciences and Technology, 2010.

[11] M. J. Swain and D. H. Ballard, Indexing via Color Hist ograms, ICCV'90, pp. 390- 393, 1990.

[12] Tat-Seng Chua, Wai-Chee Low, and Chun-Xin Chu, Relevance feedback techniques for color-based image retrieval, In Proceedings of Multi-Media Modeling'98, IEEE Computer Society, pp 24-31, 2011.

[13] M. J. Swain and D. H. Ballard, Color indexing, International Journal on Computer Vision, vol. 7, no. 1, pp. 11--32, 2011

[14] V. E. Ogle and M. Stonebraker, Chabot: retrieval from a relational database of images, IEEE Computer, vol. 28, no. 9, pp. 40-8, Sept. 1995.

[15] Michael Ortega, Yong Rui, Kaushik Chakrabarti, Sharad Mehrotra and Thomas S. Huang, Supporting Similarity Queries. In Proceeding of the ACM International Multimedia Conference, pp. 403-413, 2013. 